\title{
The Case for Fuzzy Age Boundaries in Mental Health Services
}

\author{
Anthony Jorm \\ Centre for Mental Health, Melbourne School of Population and Global Health \\ University of Melbourne, Carlton, Victoria 3010, Australia \\ Corresponding author:
}

Anthony Jorm, Centre for Mental Health, Melbourne School of Population and Global Health, University of Melbourne, 207 Bouverie Street, Carlton, Victoria 3010, Australia. Email: ajorm@unimelb.edu.au

Keywords: early intervention, youth, older person, child and adolescent

In a recent issue of the journal, Lappin et al. (2016) presented data on a 10-year follow-up of a cohort of first-episode psychosis patients. This cohort was from the United Kingdom in an era before the establishment of early intervention for psychosis services. They found that $58 \%$ of men and $71 \%$ of women had their first episode beyond the age of 25 years, which is the typical upper age limit for early intervention services in Australia. Similarly, $21 \%$ of men and $34 \%$ of women were outside the upper age limit of 35 years used by United Kingdom early intervention services. Lappin et al. (2016) concluded that current early intervention services are "gender- and age-inequitable" and argued for the extension of early intervention services to first-episode patients of any age, not just youth.

McGorry (2016) responded to this article by arguing for the benefits of youth-specific early intervention services, including transdiagnostic services that recognize the arbitrary boundaries of emerging mental disorders in youth, coupled with specialised services for young people with psychosis and other more severe mental disorders. To support people with older onsets of psychosis, McGorry argued for the creation of a separate "stream of care for genuine new onsets over 25 alongside people of the same age with illnesses whose onset was earlier but which have persisted or recurred". While essentially supporting the status quo in Australia, McGorry did concede that perhaps the upper age boundary could be increased to "closer to 30 " and that the age boundaries should be "porous and fluid".

Here I propose an approach to age-entry criteria for mental health services that has the potential to overcome the inequities of fixed age limits, yet allows a specialization on a particular developmental period of life. The proposal involves what I will call 'fuzzy' age boundaries. The term 'fuzzy' comes from a concept in mathematics called a 'fuzzy set'. A traditional set is one with sharply defined boundaries, such as the set of people who are 'teenagers'. Any person who is aged 13-19 years is in the set and all others are out. A fuzzy set is one that has grades of membership rather than clear inor-out membership. An example would be the set of people who are 'young'. A 10-year old would be clearly in, a 30-year old less so, and a 60 year-old not at all.

Figure 1 represents how the concept would apply to age boundaries of a youth mental health service. It shows on a 0 to 1 scale how eligibility for a service of a person with a mental disorder varies by age. In a traditional youth service, there are sharp-edged age boundaries, whereas in the 
fuzzy service there is a focus of specialization on a particular age group, but no sharp age-eligibility boundaries. People with mental disorders of any age would be eligible, but in practice most would come from a limited age range. Similar fuzzy age boundaries could apply to child and adolescent services and to older person services.

Below are the advantages of a fuzzy service over a traditional sharp-edged one:

1. It overcomes the age- and gender-inequities noted by Lappin et al. (2016), while still allowing for developmental specialization.

2. It allows for the fact that chronological age is an imperfect indicator of developmental stage. For example, a mature 17-year old may be suited to an adult service, while a less-mature young adult dependant on parental support might be better served by an adolescent service.

3. It allows patients with an established relationship with a particular service to stay with that service even if they cross an arbitrary upper age boundary. A person who has an episode that crosses an age boundary or who needs on-going support is unlikely to be helped by a forced move based on age criteria alone.

4. It allows patients to seek out services that best support their needs, irrespective of age. For example, a person with an early-onset dementia accompanied by behaviour problems may be best helped by an older person service, while a young adult with ADHD might find more specialized help in an adolescent service.

Could this work in practice? In fact it already does. Australia has a dual system of private practice and public mental health services. The private practice system is already fuzzy, in that practitioners can have areas of developmental specialization, but are not rigidly constrained by specific age limits. It is only the public system that is sharp edged.

Under a fuzzy system, there might be greater competition between services with overlapping developmental targets and a change in demand from some services to others. Obviously funding would need to follow the patients. If the greater competition resulted in improved patient services, this would be an important bonus of a fuzzy system.

\section{References}

Lappin JM, Heslin M, Jones PB, et al. (2016) Outcomes following first-episode psychosis-Why we should intervene early in all ages, not only youth. Australian \& New Zealand Journal of Psychiatry 50: 1055-1063.

McGorry P. (2016) Early intervention: Mission cramp versus mission creep? Australian \& New Zealand Journal of Psychiatry 50: 1033-1035. 
Figure 1. Illustration of sharp-edged versus fuzzy age boundaries to a youth mental health service

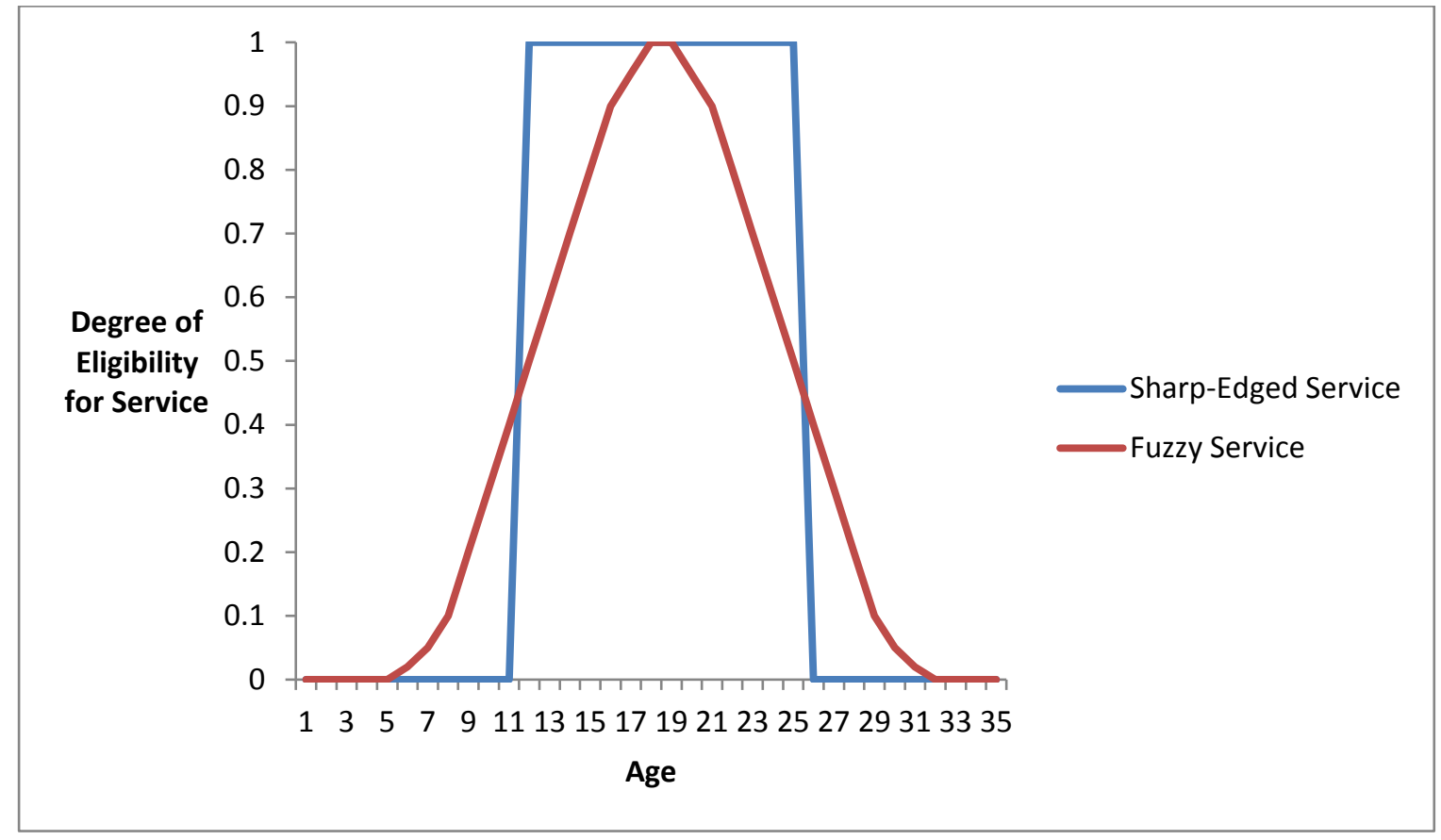

